ARTICLE

Received 25 Apr 2014 | Accepted 18 Aug 2014 | Published 24 Sep 2014

DOI: $10.1038 /$ ncomms6009

OPEN

\title{
Structural basis for biomolecular recognition in overlapping binding sites in a diiron enzyme system
}

Justin F. Acheson', Lucas J. Bailey', Nathaniel L. Elsen¹ \& Brian G. Fox ${ }^{1}$

Productive biomolecular recognition requires exquisite control of affinity and specificity. Accordingly, nature has devised many strategies to achieve proper binding interactions. Bacterial multicomponent monooxygenases provide a fascinating example, where a diiron hydroxylase must reversibly interact with both ferredoxin and catalytic effector in order to achieve electron transfer and $\mathrm{O}_{2}$ activation during catalysis. Because these two accessory proteins have distinct structures, and because the hydroxylase-effector complex covers the entire surface closest to the hydroxylase diiron centre, how ferredoxin binds to the hydroxylase has been unclear. Here we present high-resolution structures of toluene 4-monooxygenase hydroxylase complexed with its electron transfer ferredoxin and compare them with the hydroxylase-effector structure. These structures reveal that ferredoxin or effector protein binding produce different arrangements of conserved residues and customized interfaces on the hydroxylase in order to achieve different aspects of catalysis.

\footnotetext{
${ }^{1}$ Department of Biochemistry, University of Wisconsin, Biochemistry Addition, 433 Babcock Drive, Madison, Wisconsin 53706, USA. Correspondence and requests for materials should be addressed to B.G.F. (email: bgfox@biochem.wisc.edu).
} 
P rotein-protein interactions are involved in nearly all parts of life from reproduction to central metabolism to function of the global carbon cycle. The molecular contacts made in binary pairs of proteins have been elucidated and many examples are known ${ }^{1}$. However, considerably less is known about how multiple proteins can use overlapping binding surfaces ${ }^{2}$. Diiron enzymes ${ }^{3}$ provide an example where multiple protein interactions are needed to achieve catalysis. These versatile complexes are involved in many critical biological processes including hydroxylation $^{4,5}$ of the potent greenhouse gas methane ${ }^{6}$, aromatic compounds derived from lignin ${ }^{7}$ and pollutants ${ }^{8}$ and antibiotic precursors ${ }^{9}$, desaturation of fatty acids ${ }^{10,11}$ and synthesis of deoxyribonucleotides ${ }^{12,13}$.

Among the many different diiron enzyme complexes, the bacterial multicomponent monooxygenases (BMMs) have a unique requirement for both effector and redox proteins, as their absence hampers or eliminates catalysis ${ }^{4,14}$. Effector protein binding is associated with changing the redox potentials ${ }^{15}$ and spectroscopic signals from the mixed-valence and integer-spin states $^{16-20}$ of the diiron centre, improving the coupling between electron transfer and hydroxylation ${ }^{21,22}$, influencing the timing of the $\mathrm{O}_{2}$ activation steps of the catalytic cycle ${ }^{23-26}$, and altering the regio-specificity of hydroxylation ${ }^{22,27}$. Toluene 4 -monoxygenase (T4MO) is a member of these enzymes, and high-resolution structures of the hydroxylase T4moH (PDB: 3DHG), effectorhydroxylase T4moHD (PDB: 3DHH) and T4moHD-product (PDB: 3Q14) complexes are available ${ }^{28-31}$. These structures reveal conformational changes driven by $\mathrm{T} 4 \mathrm{moD}$ binding occur along the substrate access channel, within the active site, and at the diiron centre ${ }^{28}$.

In the BMMs, electrons are typically provided by an flavin adenine dinucleotide- and $[2 \mathrm{Fe}-2 \mathrm{~S}]$-containing $\mathrm{NAD}(\mathrm{P}) \mathrm{H}$ reductase $^{32}$, although direct reduction of a diiron enzyme by NADH has also been observed ${ }^{33}$. In some BMMs, an additional electron transfer ferredoxin is needed to reduce the diiron centre ${ }^{32}$. For example, in the T4MO complex, T4moC $(12 \mathrm{kDa})^{34}$ is a Riesketype ferredoxin that forms a specific complex with $\mathrm{T} 4 \mathrm{moH}^{35}$. However, structures of T4moHD show that effector protein binding covers all $\mathrm{T} 4 \mathrm{moH}$ surface residues within $\sim 20 \AA$ of the buried diiron centre in a tightly matched protein-protein interface. Therefore, how $\mathrm{T} 4 \mathrm{moH}$ forms an effective complex with $\mathrm{T} 4 \mathrm{moC}$, a protein with a different structural topology than $\mathrm{T} 4 \mathrm{moD}$, has not yet been clarified.

In this communication, we report X-ray crystal structures of the T4MO ferredoxin-hydroxylase electron-transfer complex (T4moHC) and compare them to the effector-hydroxylase $\mathrm{O}_{2}$ activation complex. The $\mathrm{T} 4 \mathrm{moHC}$ structures reveal that a combination of proximal shape complementarity, unique positioning of conserved residues and hydrogen bonds connecting the [2Fe-2S] and diiron centres, and rearrangement of a surface loop from $\mathrm{T} 4 \mathrm{moH} \sim 30 \AA$ away from the redox centres give rise to a high-specificity electron transfer complex. In contrast, the T4moHD complex uses a larger surface area and specific steric contacts to collapse the substrate access channel and place both diiron ligands and active water molecules into a unique configuration presumably poised for $\mathrm{O}_{2}$ activation. In combination, these structures provide unique new insight into the ability of protein-protein interactions to optimize configurations of conserved residues and overlapping binding interfaces to achieve different aspects of catalysis.

\section{Results}

Structure determination. To gain insight into the protein interface used for electron transfer, we determined the structure of the hydroxylase-ferredoxin complex (T4moHC). Data collection and refinement statistics for two structures are presented in Table 1 (4P1C, P $2{ }_{1} 2{ }_{1} 2_{1}$ space group, $2.4 \AA$; $4 \mathrm{P} 1 \mathrm{~B}, \mathrm{P} 3{ }_{2} 21$, $2.05 \AA$ ). In both diffraction data sets, the asymmetric unit contains the biological dimer, with the TmoA, TmoB, TmoE and TmoC polypeptides (ABEC protomer) arranged into an $(\mathrm{ABEC})_{2}$ structure. All polypeptide chains, diiron centres and [2Fe-2S] centres have full occupancy. The two structures have minor differences in resolution, variant of $\mathrm{T} 4 \mathrm{moC}$ used and whether acetate was present in the crystallization buffer. Further details of the structure determination can be found in Methods.

Differences between 4P1C and 4P1B. T4moC used to obtain the lower resolution $4 \mathrm{P} 1 \mathrm{C}$ structure $\left(\mathrm{P} 2_{1} 2_{1} 2_{1}\right.$ space group, $2.4 \AA$ resolution) contains three mutations and acetate was not present in the crystallization buffers. The combination of Cys84Ala and Cys85Ala previously yielded a $1.4 \AA$ structure of $\mathrm{T} 4 \mathrm{moC}^{36}$; the third mutation, Cys7Ser, was created as part of this work to remove the remaining surface cysteine in order to eliminate possibilities for intermolecular disulfide bond formation. 4P1C contains no mutations in the biologically relevant $\mathrm{HC}$ interface. In the $4 \mathrm{P} 1 \mathrm{C}$ active site, a polyethylene glycol (PEG) molecule has been modelled as a weakly coordinating $\mu$-alkoxo ligand extending from the diiron centre into the substrate-binding cavity. Similar partially complete electron density assigned to exogenous ligands has been observed in other diiron hydroxylase structures 37

T4moC used to obtain the higher resolution $4 \mathrm{P} 1 \mathrm{~B}$ structure (P3 $21,2.05 \AA$ resolution) contained an additional Glu16Cys mutation to potentially allow covalent crosslinking to mutated

Table 1 | Data collection and refinement statistics.

\begin{tabular}{|c|c|c|}
\hline & 4P1C & 4P1B \\
\hline \multicolumn{3}{|l|}{ Data collection } \\
\hline Space group & $\mathrm{P} 22_{1} 2_{1} 2_{1}$ & $\mathrm{P}_{2} 21$ \\
\hline \multicolumn{3}{|l|}{ Cell dimensions } \\
\hline$a, b, c(\AA)$ & $95.22,106.35,213.42$ & $128.37,128.37,284.46$ \\
\hline$\alpha, \beta, \gamma\left({ }^{\circ}\right)$ & $90,90,90$ & $90,90,120$ \\
\hline \multirow[t]{2}{*}{ Resolution $(\AA)$} & $47.69-2.40$ & $42.56-2.05$ \\
\hline & $(2.43-2.40)^{\star}$ & $(2.07-2.05)$ \\
\hline$R_{\text {sym }}$ & $0.129(0.76)$ & $0.118(0.65)$ \\
\hline$|/ \sigma|$ & $12.4(2.0)$ & $6.6(2.2)$ \\
\hline Completeness (\%) & $95.10(93.00)$ & $100(100)$ \\
\hline Redundancy & $6.0(4.5)$ & $8.4(7.6)$ \\
\hline \multicolumn{3}{|l|}{ Refinement } \\
\hline Resolution $(\AA)$ & $47.69-2.40$ & $42.56-2.06$ \\
\hline No. of reflections & $81,284 / 6649$ & $188,039 / 12,518$ \\
\hline$R_{\text {work }} / R_{\text {free }}$ & $0.153 / 0.215$ & $0.146 / 0.177$ \\
\hline \multicolumn{3}{|l|}{ No. of atoms } \\
\hline Protein & 16,137 & 16,269 \\
\hline Ligand/ion & 24 & 66 \\
\hline Water & 609 & 1,917 \\
\hline \multicolumn{3}{|l|}{$B$-factors } \\
\hline Protein & 20.17 & 28.87 \\
\hline Ligand/ion & 21.36 & 39.71 \\
\hline Water & 19.92 & 38.08 \\
\hline \multicolumn{3}{|l|}{ R.m.s deviations } \\
\hline Bond lengths $(\AA)$ & 0.008 & 0.008 \\
\hline Bond angles $\left(^{\circ}\right)$ & 1.077 & 0.859 \\
\hline
\end{tabular}


variants of $\mathrm{T} 4 \mathrm{moH}$, however, crystals were obtained without the use of chemical cross-linkers. This mutation resulted in a loss of a hydrogen bond between $\mathrm{T} 4 \mathrm{moC}$ and $\mathrm{TmoE}$, however, the position of binding of $\mathrm{T} 4 \mathrm{moC}$ was nearly indistinguishable to that of $4 \mathrm{P} 1 \mathrm{C}$. In the $4 \mathrm{P} 1 \mathrm{~B}$ complex, acetate was included in the crystallization solutions, and yielded an acetate bound to the diiron centre as is often seen in other diiron enzymes and $\mathrm{BMMs}^{38}$. Root mean squared deviation (RMSD) comparisons of subunits in the two $\mathrm{T} 4 \mathrm{moHC}$ structures are shown in Supplementary Table 1.

Binding interface. T4moC binds in the canyon formed at the dimer interface of $\mathrm{T} 4 \mathrm{moH}$, making extensive contacts with TmoA (Fig. 1a and Supplementary Fig. 1a). Furthermore, T4moC bridges to TmoE of the opposite protomer of $\mathrm{T} 4 \mathrm{moH}$ to recognize the quaternary structure of the hydroxylase. The T4moHC complex utilizes a smaller protein-protein interface with an area of $\sim 1150 \AA^{2}$ and the interface is dominated by electrostatic interactions (Fig. 2a and Supplementary Fig. 1a). There are 23 residues from TmoA within $5 \AA$ of $\mathrm{T} 4 \mathrm{moC} ; 12$ are also within $15 \AA$ of the diiron centre of TmoA. Six deeply recessed residues, including diiron centre ligand Glu231, are uniquely involved in the T4moHC complex. Consequently, the T4moHC interface includes all TmoA residues that provide closest approach from the surface to the diiron centre.

For comparison, the T4moHD interface $\left(\sim 2,200 \AA^{2}\right)$ is $\sim 2 \times$ larger than the $\mathrm{T} 4 \mathrm{moHC}$ interface (Fig. 2b). In T4moHD, 53 residues from TmoA are within $5 \AA$ of $\mathrm{T} 4 \mathrm{moD}$, including both polar and non-polar residues (Fig. $2 \mathrm{~b}$ and Supplementary Fig. 1b). Seventeen of these residues are also found in the T4moHC interface; however, a water-filled cavity overlays the six most deeply recessed residues from TmoA in the T4moHD complex.

Inhibition by $\mathbf{T} 4 \mathrm{moD}$. T4moD activates toluene hydroxylation at low concentrations and inhibits at high concentrations ${ }^{22}$. Kinetic studies show that $\mathrm{T} 4 \mathrm{moD}$ acts as a competitive inhibitor of T4moC by increasing the apparent $K_{\mathrm{M}}(\sim 3 \mu \mathrm{M}$ increased to $16 \mu \mathrm{M})$ while yielding the same $k_{\text {cat }}\left(\sim 3 \mathrm{~s}^{-1}\right.$ per $\left.\mathrm{T} 4 \mathrm{moH}\right)$ at saturating T4moC (Supplementary Table 2 and Supplementary Fig. 2a). The $K_{\mathrm{I}}$ for $\mathrm{T} 4 \mathrm{moD}$ was also increased from $45 \pm 7$ to $240 \pm 50 \mu \mathrm{M}$ when [T4moC] was increased from 12 to $42 \mu \mathrm{M}$ (Supplementary Table 2 and Supplementary Fig. 2b). These steady-state kinetic behaviours are consistent with the overlap in binding sites established by the structures of $\mathrm{T} 4 \mathrm{moHC}$ and T4moHD (Fig. 1).

Substrate channels. As first shown in toluene/o-xylene monooxygenase $^{39}, \mathrm{~T} 4 \mathrm{moH}$ has a hydrophobic tunnel with a forked entrance that allows access to the active site ${ }^{28}$. T4moC binding leaves this tunnel open (Fig. 2c) and in a nearly identical configuration to $\mathrm{T} 4 \mathrm{moH}$. With the tunnel open, substrates may enter and product may leave through the forked position controlled by residues L208 and D211 (Fig. 2c). T4moD binding shifts helix E (Fig. 2d), which moves these gating residues and collapses the active site access at the fork position.

Distal contribution to T4moC binding. The T4moHC structure revealed that $\mathrm{T} 4 \mathrm{moC}$ bridges between (ABE) protomers in $\mathrm{T} 4 \mathrm{moH}$. Loop residues 10-21 (RTWSHLAEMRKK) from the opposite TmoE make electrostatic contacts with $\mathrm{T} 4 \mathrm{moC}$ and also form a hydrophobic loop surrounding $\mathrm{T} 4 \mathrm{moC}$ Trp13 (Fig. 3a). Thus, molecular recognition of the ferredoxin is enhanced through interaction with the hydroxylase over $30 \AA$ distal from the primary interface. The TmoE loop
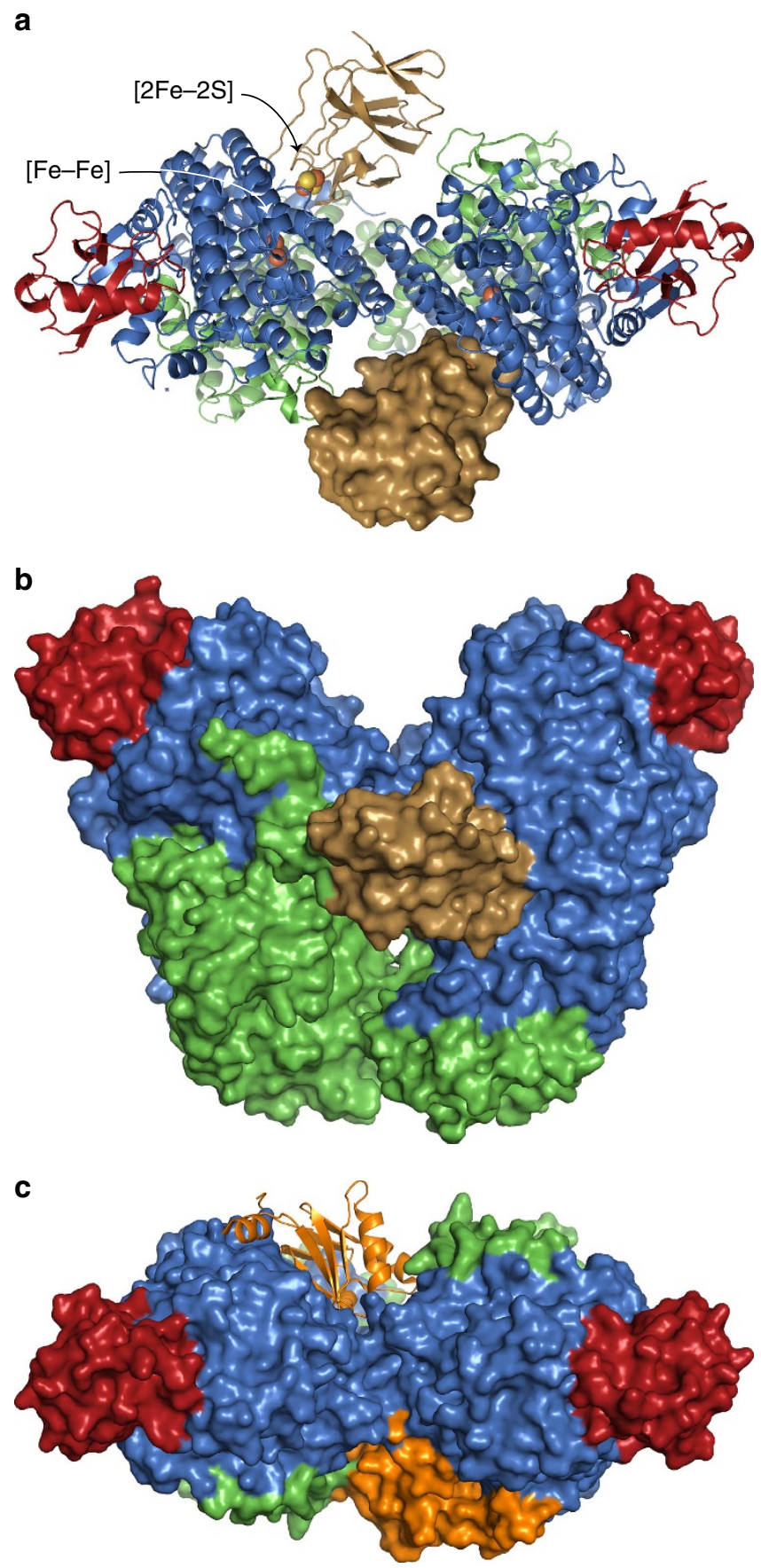

Figure 1 | Structure of T4moHC complex. The T4moHC complex consists of two copies of the TmoA (blue), TmoB (red), TmoE (green) and Riesketype ferredoxin $\mathrm{T} 4 \mathrm{moC}$ (gold) polypeptides. (a,b) Two views given by $90^{\circ}$ rotation about the $x$ axis. The positions of the [2Fe-2S] cluster in $\mathrm{TmoC}$, and the position of the diiron centre [Fe-Fe] in TmoA are shown as spheres in a to illustrate their relative positions in the complex. (c) T4moD (orange) is shown bound to the same surface of TmoA as T4moC.

residues adopt different configurations in both the uncomplexed $\mathrm{T} 4 \mathrm{moH}$ and the T4moHD complex structures (Supplementary Fig. 4). In the $\mathrm{T} 4 \mathrm{moH}$ crystals (PBD 3DHG), the loop makes several contacts with an adjacent dimer in the crystal lattice. In solution, however, the TmoE loop would be solvent exposed and likely disordered or able to sample multiple conformations ${ }^{40}$. When T4moD binds (PDB 3DHH), this loop adopts a third conformation as a result of minor binding interactions with the effector (Fig. 3a and Supplementary Fig. 4). 
a

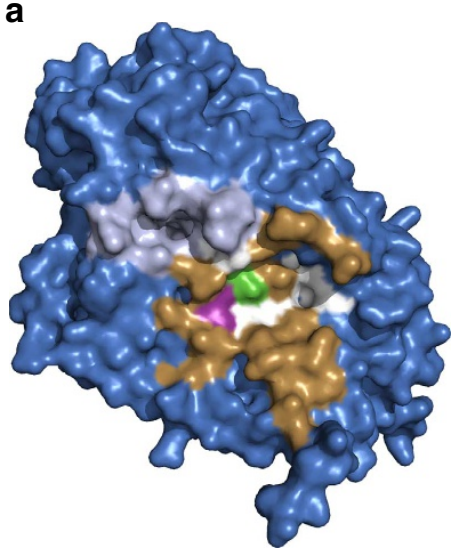

C

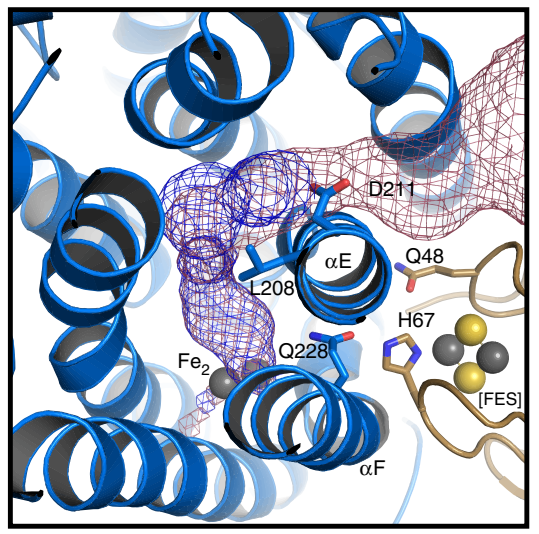

b

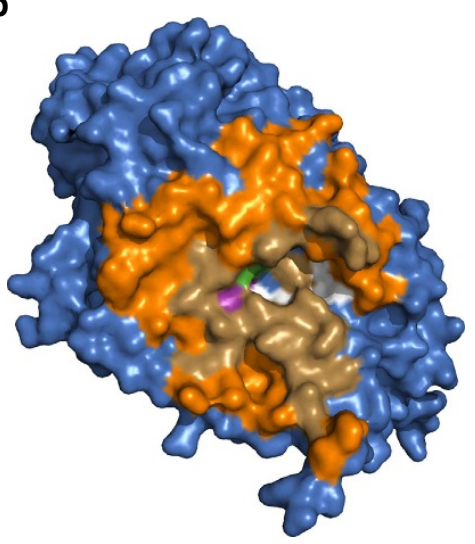

d

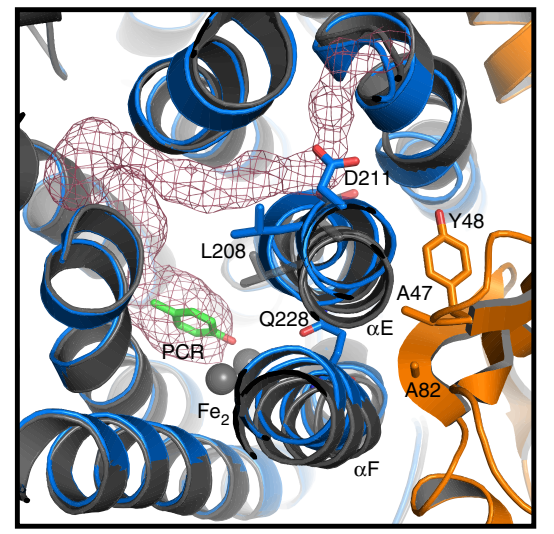

Figure 2 | Surfaces and tunnels of TmoA. (a) Surface of TmoA (blue) alone, showing residues involved in T4moC binding (gold), entrance to the substrate channel (light blue), residues Asn202 (green) and Gln228 (purple), and six residues unique to the T4moHC interface (white). (b) Surface of TmoA from the T4moHD complex, showing 53 residues within $5 \AA$ of $T 4 \mathrm{moD}$ that are unique to the $T 4 \mathrm{moHD}$ complex (orange), 17 residues in common with the $\mathrm{T} 4 \mathrm{moHC}$ complex (gold), and six residues unique to the $\mathrm{T} 4 \mathrm{moHC}$ complex (white). (c) Mesh mapping (raspberry and blue) of the open tunnel from solvent to the active site in TmoA from the T4moHC complex. (d) Mesh mapping of the closed tunnel in the T4moHD complex caused by repositioning of TmoA residues Leu208 and Asp211 relative to their positions in T4moHC (dark grey). These shifts are produced by steric contacts with $\mathrm{T} 4 \mathrm{moD}$ residues Ala47 and Tyr48, causing complete collapse of the entrance to the substrate channel at the fork position.

Electron transfer. The distance between redox partners is an important factor in efficient electron transfer. In the T4moHC complex, the distance between the [2Fe-2S] cofactor and diiron centre is $\sim 12 \AA$ (Fig. 3b and Supplementary Fig. 5), a typical distance compared with other biological electron transfer complexes $^{41,42}$. HARLEM predicts a shortest gap $3.4 \AA$ electron transfer (Fig. 3b) between His67 of T4moC and Glu231 of TmoA, which are ligands to the [2Fe-2S] and diiron centres, respectively ${ }^{43}$. A hydrogen-bonding network, which is generated in the protein interface, connects residues His67 of T4moC to Gln228 of TmoA and then to Fe2 ligand Glu231.

Outer sphere active site residues. A triad of non-ligand TmoA residues, Thr201, Asn202 and Gln228, are important in catalysis $^{28,29,44}$. The involvement of these residues in positioning active site water is consistent for both T4moHC and T4moHD (Fig. 3b,c), but with several differences revealed by the structures. Relative to their positions in T4moHD (Fig. 3c), Thr201 is shifted by $\sim 2 \AA$ inside the T4moHC active site (Fig. 3b), whereas Asn202 and Gln228 are displaced by $\sim 3 \AA$ and $\sim 2 \AA$, respectively, in order to accommodate His67 of T4moC, a ligand to the $[2 \mathrm{Fe}-2 \mathrm{~S}]$ cofactor. Along with the carbonyl oxygen of $\mathrm{Fe} 2$ ligand Glu197, this triad holds $\mathrm{HOH} 506 \sim 4 \AA$ from $\mathrm{Fe} 2$ (Fig. 3b). Importantly, Glu231 in T4moHC has bidentate coordination to $\mathrm{Fe} 2$, thus cannot provide hydrogen bonds to either Thr201 or an active site water molecule, which are both observed in the T4moHD complex.

No waters are present in $\mathrm{T} 4 \mathrm{moHC}$ at a position comparable to where HOH519 and HOH507 are found in the T4moHD and reduced T4moHD complexes, respectively (Figs 3b,c and 4$)^{28,29}$. We have hypothesized that the uniquely positioned water in T4moHD is a potential candidate for proton donation during catalysis, perhaps to facilitate cleavage of the $\mathrm{O}-\mathrm{O}$ bond in a peroxo-diferric complex ${ }^{30}$, or to maintain charge balance as the $\mathrm{p} K_{\mathrm{a}}$ of metal-bound water/hydroxide responds to changes in redox state of the diiron centre.

Active site coordination. The $\mathrm{T} 4 \mathrm{moHC}$ complex has a unique active site geometry compared with other $\mathrm{T} 4 \mathrm{moH}$ structures (Fig. 4 and Supplementary Fig. 6). With the exceptions of Glu104 and Glu231, other diiron centre ligands have nearly identical geometries to those observed previously. In the higher-resolution 4P1B structure, Glu104 is present in two equal-occupancy configurations with the carboxyl groups rotated $\sim 90^{\circ}$ relative to each other. Acetate is also observed as a bridging ligand to the diiron centre. Glu231 was modelled in a single, bidentate configuration that is similar to the geometry of the homologous Glu243 observed in crystals of mixed valence methane 
$\mathbf{a}$

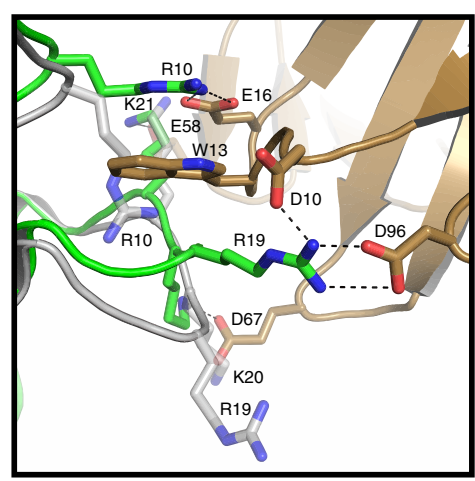

b

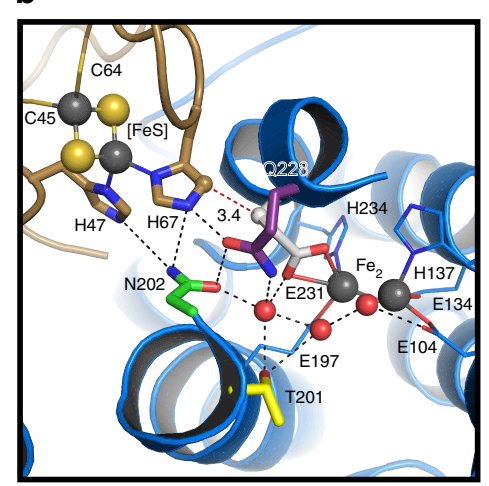

C

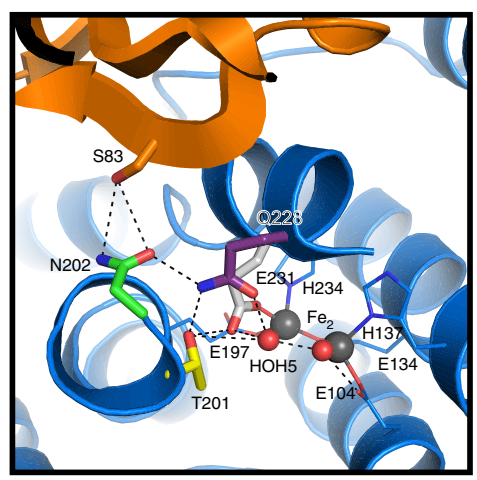

Figure 3 | Effects of T4moC and T4moD binding to T4moH. (a) Binding site for T4moC Trp13 formed from TmoE residues 10-21 (green cartoon with sticks shown for Arg10 and Arg19 that provide stabilizing $\mathrm{H}$-bonding interactions with T4moC Glu16, Asp10 and Asp96). Another view of this interface shows the positions of the TmoE loop residues in the T4moHD complex (PDB accession 3DHH) are shown in Supplementary Fig. 3. (b) Electron transfer interface in T4moHC including T4moC (gold) and T4moH diiron ligands (blue), E231 (white), Asn202 (green) and Gln228 (purple). The distance from the $[2 \mathrm{Fe}-2 \mathrm{~S}]$ to the diiron centre is $\sim 12 \AA$, and the closest contact between T4moC His 67 and TmoA Glu231 is indicated by a dashed red line (3.4 $\AA$ ). (c) T4moD binding and hydrogen bonding rearrangement poises a catalytic water $\mathrm{HOH} 519$ for $\mathrm{O}_{2}$ activation.

monooxygenase ${ }^{45}$. This configuration is distinct from the monodentate geometry observed in resting $\mathrm{T} 4 \mathrm{moH}$ (PDB $3 \mathrm{DHG}$, Fig. 4a) other diferric $\mathrm{BMMs}^{38,39,46}$ and the bidentate carboxylate-bridging geometry in reduced T4moHD (PDB 3DHI, Fig. 4d) and other BMMs ${ }^{45}$.

\section{Discussion}

The T4moHC complex reveals, to the best of our knowledge, the first structural account of how overlapped binding sites can be shared in a multicomponent enzyme. The combination of shape complementarity, unique configurations of three conserved residues and their hydrogen bonds in the interfaces used for either electron transfer or $\mathrm{O}_{2}$ activation, and a distal, bindinginduced rearrangement are used to achieve both high specificity and efficiency of the enzyme reaction with toluene.

Shape complementarity in the deepest portion of the binding interface allows the ferredoxin [2Fe-2S] centre to achieve the closest possible through-space approach to the hydroxylase diiron active site, whereas the unique 4-,5-coordinate geometry produced by $\mathrm{T} 4 \mathrm{moC}$ binding (Fig. 4c) likely represents a new diiron centre configuration involved in electron transfer. In the outer portion of the overlapping binding surface, rearrangements of alpha helices on the hydroxylase place a triad of conserved hydroxylase residues (Thr201, Asn202, Gln228) into a hydrogenbonded electron transfer interface that connects the [2Fe-2S] and diiron centres and entrains a water molecule that may help to maintain charge balance during electron transfer. This triad has a different arrangement in the hydroxylase-effector complex ${ }^{28}$, which plausibly serves to position diiron ligand Glu231 and active site water for $\mathrm{O}_{2}$ activation, proton transfer and substrate hydroxylation steps of the catalytic cycle ${ }^{8,14,22,26}$.

Figure 4 shows that Glu231 has a different coordination geometry in $\mathrm{T} 4 \mathrm{moH}$ (Fig. 4a, resting diferric state), T4moHD (Fig. 4c, diferric state) and reduced T4moHD (Fig. 4d, diferrous state produced in the presence of excess sodium dithionite). Thus, changes in $\mathrm{T} 4 \mathrm{moH}$ coordination are produced both by protein binding and by changes in redox state of the diiron centre. The bidentate geometry of Glu231 in T4moHC is most similar to the coordination observed for homologous Glu243 in methane monooxygenase crystals treated with redox mediators so as to stabilize a $1 \mathrm{e}^{-}$reduced, mixed valence state $\mathrm{e}^{45}$. In the absence of spectroscopic evidence obtained on T4moHC crystals, assignment of the redox state of a diiron centre in a crystal is an inferential process. Because ferredoxins are known to be reduced by synchrotron radiation ${ }^{47}$, it is possible that $\mathrm{T} 4 \mathrm{moHC}$ contains either a diferric or a mixed valence diiron centre, but less likely a diferrous centre because the bidentate carboxylatebridging geometry is not present.

Acetate has been frequently used as an additive in the crystallization of diiron enzymes ${ }^{28,38}$. In $4 \mathrm{P} 1 \mathrm{~B}$, acetate formed a bridge between $\mathrm{Fel}$ and $\mathrm{Fe} 2$, with the methyl group uniquely pointing towards TmoA Gln228 (Fig. 4c). In other diiron enzyme structures, Fe-bound acetate projects away from the diiron centre into the substrate binding cavity, a $\sim 90^{\circ}$ reorientation relative to that observed in 4P1B. Also in 4P1B, Glu104 showed two conformations with occupancies of 61/39 in chain A and 53/47 in chain $\mathrm{D}$. The majority conformation was hydrogen bonded to the bound acetate, whereas the other conformation had the carboxylate rotated $90^{\circ}$ towards the substrate-binding cavity with respect to previous structures. In the rotated conformation, the carboxylate group was hydrogen bonded to $\mathrm{HOH} 966$ that also weakly interacted with Fe1 (2.7 $\AA$ ) and Fe2 (3.3 $\AA$ ). These new configurations further demonstrate the influence of ferredoxin binding on the properties of the diiron centre.

Control of substrate access is presumed necessary to promote coupling of diiron centre reduction with substrate hydroxylation $^{31,39}$. Binding of T4moD shifts helix E (Fig. 2d), effectively trapping substrate in the active site and deterring uncoupled electron consumption. Release of product from the diferric centre may be facilitated by reduction and the open egress in $\mathrm{T}_{4} \mathrm{moHC}^{31}$. Consequently, the T4moHC complex enables both access and egress to the active site and also electron transfer, whereas the T4moHD complex restricts access to the active site but poises the complex for catalysis by repositioning the diiron centre ligands and active site waters ${ }^{28,31}$. The requirement for separate effector and redox partners to control active site access, provide electron transfer and rearrange the position of conserved active residues for catalysis distinguishes the BMMs from P450s, which use only a ferredoxin to achieve both effector and redox functions without major conformational changes ${ }^{48}$.

Previous studies with other Rieske-type ferredoxins showed that $\mathrm{TbuB}$, the Rieske-type ferredoxin from Ralstonia pickettii toluene monooxygenase ( $46 \%$ sequence identity, Swissmodel ${ }^{49}$, QMEAN score-0.2), was able to substitute as the proximal electron donor for $\mathrm{T} 4 \mathrm{moC}$ in catalysis, whereas $\mathrm{BphF}$ from 

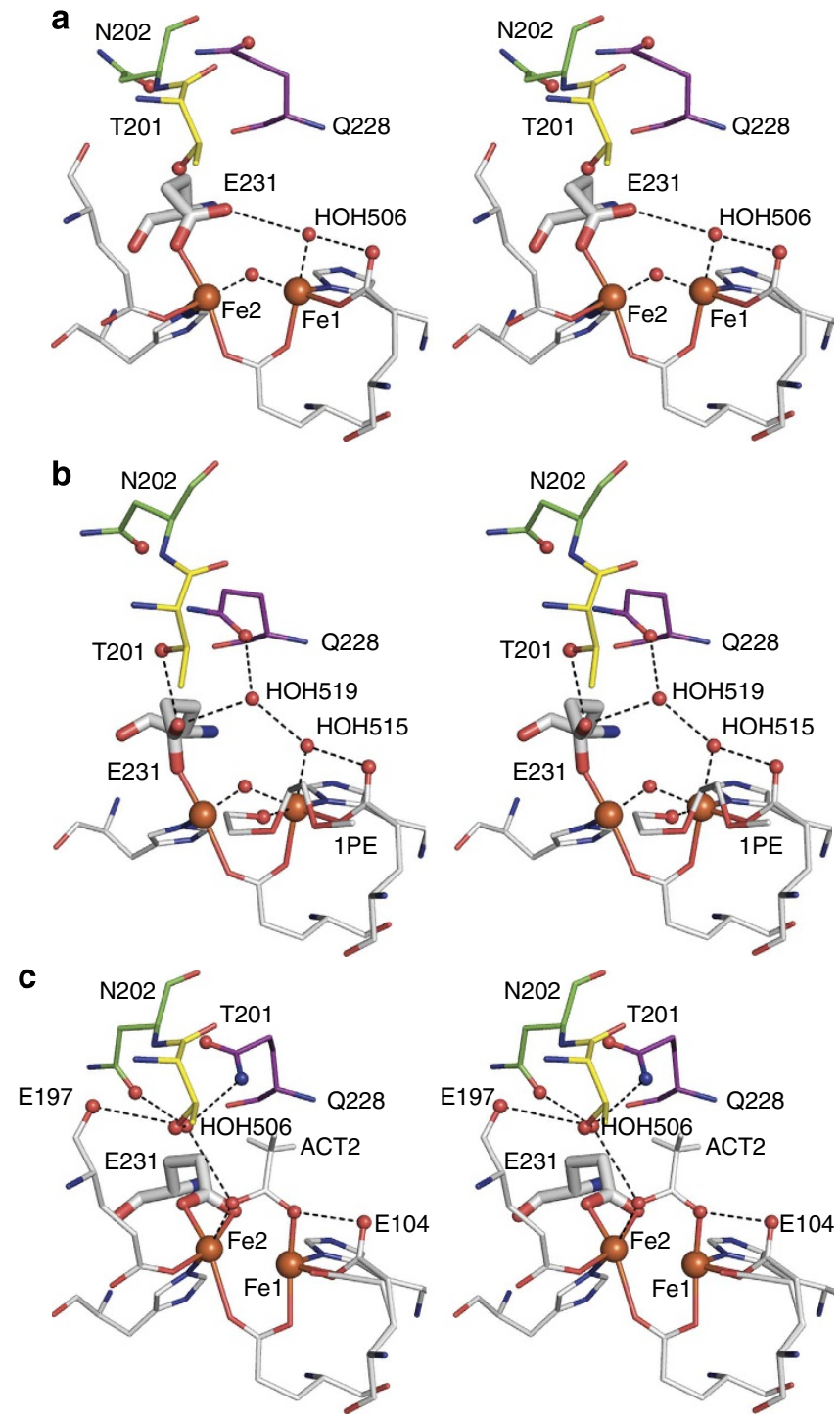

d

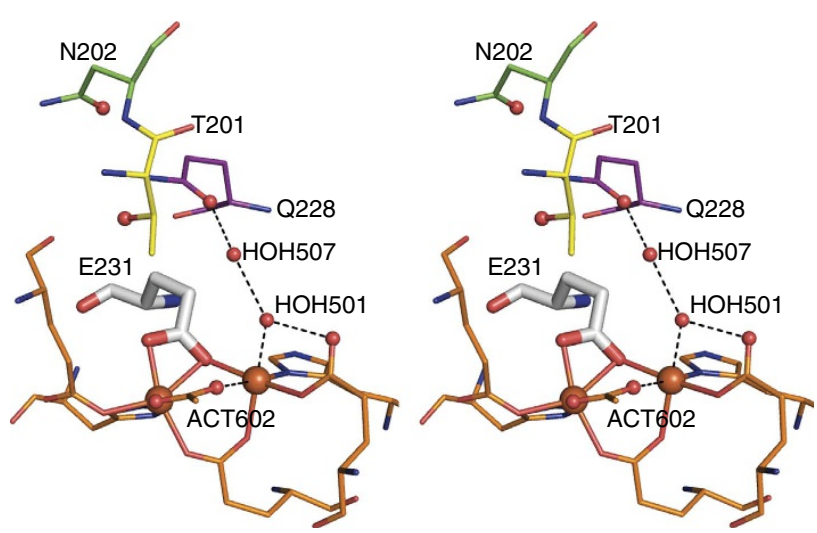

Figure 4 | Stereo diiron centre configurations in different complexes and redox states of T4moH. Positions of key residues Thr201 (yellow), Asn202 (green), Gln228 (purple), Glu231 (white) shown as sticks. Unique active site water molecules are identified by their PDB ID numbers. (a) Resting $T 4 \mathrm{moH}$ (PDB 3DHG) with diferric centre and iron atoms Fe1 and Fe2 identified. (b) Resting T4moHD (PDB 3DHH) with diferric centre and polyethylene glycol (1PE) bound to $\mathrm{Fe} 1$ and projecting into the substrate cavity. (c) Resting $\mathrm{T} 4 \mathrm{moHC}$ (PDB 4P1B) with acetate (ACT2) bound in a bridging position between Fe2 and Fe1. (d) Reduced T4moHD (PDB 3DHI) with acetate (ACT605) bound in a bridging position that projects into the substrate cavity.
Burkeholderia cepacia LB400 biphenyl dioxygenase (26\% sequence identity, $0.8 \AA \mathrm{rms}$ deviation, PDB $1 \mathrm{FQT})$ could not ${ }^{35,50}$. The present structure provides insight into these prior results. In a homology model with $\mathrm{T} 4 \mathrm{moC}$, TbuB has identical residues in nearly every interface position, including $\mathrm{T} 4 \mathrm{moC} \mathrm{Trp} 13$ that interacts with the adjacent protomer. Furthermore, substitution of T4moC Val14 by TbuB Glu14 may allow formation of an additional salt bridge with TmoE Lys20 on the adjacent protomer. With this similarity, it is likely the interactions with the flexible loop from TmoE observed in T4moHC will also occur with TbuB. Minor differences include the substitution of T4moC Asp83 with TbuB Gly82, resulting in the loss of an electrostatic interaction, and two fewer residues at the $\mathrm{C}$-terminus, leading to a loss of favourable packing interactions with TmoA Pro50 and TmoA Gln336. These changes may account for the modest increase in apparent $K_{\mathrm{M}}$ for TbuB. In contrast, BphF is missing a looporganizing hydrophobic residue analogous to $\mathrm{T} 4 \mathrm{moC} \mathrm{Trp} 13$, and so may not induce the stabilizing disorder-to-order transition. BphF also has a loop containing three Pro residues, which would unfavourably protrude into the electron transfer interface at TmoA Tyr51. The loss of key interactions that help to anchor the ferredoxin and likelihood of steric clashes in the interface provides a structural basis for inability of BphF to act as an effective reductant for $\mathrm{T} 4 \mathrm{moH}$.

The functional imperative to bind structurally distinct proteins to the same docking surface presents an interesting dilemma in biomolecular specificity. In methane monooxygenase, effector protein binding advances the catalytic cycle by enhancing the rate of formation of several increasingly oxidized intermediates (diferrous- $\mathrm{O}_{2}$ adduct $\left(\mathrm{P}^{*}\right)^{26}$; diferric peroxo $(\mathrm{P})^{51}$; and diferryl $(\mathrm{Q})^{51}$ ) formed after combination of the diferrous centre with $\mathrm{O}_{2}$. However, reduction of the diferric hydroxylase to the diferrous state, which is required to initiate the catalytic cycle, also apparently requires disassociation of the tightly bound effector $\left(K_{\mathrm{D}}=70 \mathrm{nM}\right.$ (ref. 24) so that the ferredoxin (reductase) may bind. In T4MO, the overlapped binding sites for effector and ferredoxin with hydroxylase may be a key element in redox gating of the catalytic cycle, with formation of diferrous centre controlled by competitive formation of either a non-productive diferric T4moHD complex or a complex with $1 \mathrm{e}^{-}$reduced $\mathrm{T} 4 \mathrm{moC}$ at the same surface of $\mathrm{T} 4 \mathrm{moH}$ and involving the same conserved residues. Preferential reduction to the diferrous state may arise from an $\sim 10^{4}$-fold weaker affinity of the effector as the hydroxylase is reduced ${ }^{52}$, which would otherwise block reduction at the overlapping sites, and by an increase in the redox potential of the diiron centre correlated with rearrangements of the diiron centre ligands. Subsequent combination of the diferrous centre with $\mathrm{O}_{2}$ would initiate the oxidative steps of catalysis, and an increase in the affinity of effector would productively drive the catalytic cycle forward ${ }^{24}$. Presence of a tightly bound effector during this stage of catalysis would also help to prevent adventitious reduction of the activated intermediates ${ }^{21,53}$.

Here we have shown how binding of either effector or ferredoxin to an overlapping surface on the diiron hydroxylase can be achieved. Although effector protein binds tightly to a large surface area and induces a conformational change extending into the hydroxylase active site $^{28}$, ferredoxin binding combines rearrangement of a loop $\sim 30 \AA$ distal with a tightly matched, hydrogen bonded contact surface that places the [2Fe-2S] and diiron centres in their closest possible approach for electron transfer. The differences provided by a combination of intricate shape matching, alternative conformations of a conserved triad of residues and flexible accommodation provides an exquisite example of how specificity can be achieved in biomolecular catalysis. 


\section{Methods}

Cloning. All mutagenesis experiments were carried out by Quickchange (Stratagene) and sequence-verified at the University of Wisconsin Biotechnology Center. Vector pVP58kABE (T4moH) was modified to contain stop codons at codon 492 in the tmoA gene and codon 307 in $t m o E$ gene in order to eliminate C-terminal sequences that were disordered in all prior crystal structures. Vector pET28T4moCD, encoding C84A/C85A mutations in T4moC and also T4moD, was modified to also encode an E16C mutation in T4moC. Both the pET28T4moCD and pET28T4moCD E16C plasmids were modified to encode a C7S mutation to remove the only remaining surface cysteine from $\mathrm{T} 4 \mathrm{moC}$.

Protein expression. pVP58kABE was transformed into Escherichia coli BL21 and the T4moCD vectors were transformed into $E$. coli $\mathrm{BL} 21(\mathrm{DE} 3)$. The transformation reaction was plated onto Luria-Bertani agar plates containing $50 \mu \mathrm{g} \mathrm{ml}^{-1}$ of kanamycin and grown overnight at $37^{\circ} \mathrm{C}$. A starting inoculum for the stirred vessel fermentation was prepared by placing a single colony into $2 \mathrm{ml}$ of non-inducing medium containing $\left(\mathrm{NH}_{4}\right)_{2} \mathrm{SO}_{4}, \mathrm{KH}_{2} \mathrm{PO}_{4}, \mathrm{Na}_{2} \mathrm{HPO}_{4}$, aspartate, amino acids, and glucose and $50 \mathrm{\mu g} \mathrm{ml}^{-1}$ of kanamycin ${ }^{54}$. The $2-\mathrm{ml}$ culture was incubated at $37^{\circ} \mathrm{C}$ with shaking at 250 r.p.m. until it reached an $\mathrm{OD}_{600}$ between 0.5 and 1 . Next, two 21 flasks were prepared containing $500 \mathrm{ml}$ of the same medium and $50 \mu \mathrm{g} \mathrm{ml}^{-1}$ kanamycin was inoculated with $\sim 500 \mu \mathrm{l}$ of the $2 \mathrm{ml}$ culture and incubated overnight ( $\sim 12$ to $14 \mathrm{~h}$ ) at $25^{\circ} \mathrm{C}$ with shaking at 250 r.p.m. When the overnight cultures had reached an $\mathrm{OD}_{600} \sim 1$, they we added to 91 of medium in a Bioflo 110 fermenter (New Brunswick Scientific) and grown at $37^{\circ} \mathrm{C}$ with agitation at 300 r.p.m. The dissolved $\mathrm{O}_{2}$ content was allowed to decrease to $20 \%$ of air saturation and subsequently, the agitation was allowed to increase under feedback control to maintain the dissolved $\mathrm{O}_{2}$ content at $20 \%$ of air saturation. At an $\mathrm{OD}_{600}$ $\sim 3$, the temperature was decreased to $25^{\circ} \mathrm{C}$ and $0.4 \mathrm{~g}$ of isopropyl- $\beta$-Dthiogalactoside (Fisher Scientific) dissolved in $10 \mathrm{ml}$ of $\mathrm{ddH}_{2} \mathrm{O}, 20 \mathrm{~g}$ of Casamino acids (Fisher Scientific) and $36 \mathrm{~g}$ of lactose were added to the culture medium. After induction of protein expression, the culture growth was continued for $5 \mathrm{~h}$, and the $\mathrm{OD}_{600}$ reached $\sim 8$. The cells were recovered by centrifugation at $4,200 \mathrm{~g}$ for $25 \mathrm{~min}$ at $4{ }^{\circ} \mathrm{C}$ in a JS-4.2 rotor and an AvantiTM J-HC centrifuge (Beckman Coulter). The yield from this expression protocol was $\sim 8$ to $9 \mathrm{~g}$ of wet cell paste per litre of culture medium. The cell paste was stored at $-80^{\circ} \mathrm{C}$.

Purification of T4moH. T4moH cell paste was re-suspended in $25 \mathrm{mM}$ MOPS, pH 6.9 , containing $150 \mathrm{mM} \mathrm{NaCl}$ and $2 \%$ glycerol at a ratio of $1.5 \mathrm{ml}$ per g of wet cell paste $^{31}$. The cell suspension was sonicated on ice at high intensity for $8 \mathrm{~min}(15 \mathrm{~s}$ on; $30 \mathrm{~s}$ off). The supernatant from the sonicated cells was recovered by centrifugation at $39,200 \mathrm{~g}$ for $60 \mathrm{~min}$ at $4^{\circ} \mathrm{C}$. The supernatant was carefully decanted and diluted with two volumes of the above buffer and loaded onto a DEAE Sepharose column ( $45 \mathrm{~mm}$ diameter $\times 250 \mathrm{~mm}$, GE Health Care) equilibrated in the above buffer and eluted in a $150-450 \mathrm{mM} \mathrm{NaCl}$ gradient in the same buffer at a linear flow rate of $40 \mathrm{~cm} \mathrm{~h}^{-1}$. Fractions were pooled based on both activity and purity as determined by SDS-polyacrylamide gel electrophoresis (SDS-PAGE. Pooled fractions were concentrated and applied to a Sephacryl S-300 (45 mm diameter $\times 1,000 \mathrm{~mm}$, GE Health Care) column equilibrated in $25 \mathrm{mM}$ MOPS, pH 7.5, $200 \mathrm{mM} \mathrm{NaCl}$ and $5 \%$ glycerol at a flow rate of $5 \mathrm{ml} \mathrm{min}^{-1}$. Fractions were pooled based on activity and purity, concentrated to $\sim 800 \mu \mathrm{M}$ active sites, and exchanged into $10 \mathrm{mM}$ MOPS, pH 6.9, containing $200 \mathrm{mM} \mathrm{NaCl}$. The purified protein was drop frozen in liquid $\mathrm{N}_{2}$ and stored at $-80^{\circ} \mathrm{C}$.

Purification of T4moC and T4moD. Cell paste containing a co-expression of $\mathrm{T} 4 \mathrm{moC}$ and $\mathrm{T} 4 \mathrm{moD}$ was re-suspended in $25 \mathrm{mM}$ MOPS, pH 7.5 , containing $2 \%$ glycerol but no $\mathrm{NaCl}$ at a ratio of $1.5 \mathrm{ml}$ per $\mathrm{g}$ of wet cell paste ${ }^{31}$. The cell-free extract was prepared as described above for T4moH. The supernatant was carefully decanted and diluted with two volumes of the above buffer and loaded onto a DEAE Sepharose column $(45 \mathrm{~mm}$ diameter $\times 250 \mathrm{~mm}$, GE Health Care) equilibrated in the above buffer and eluted in a $0-450 \mathrm{mM} \mathrm{NaCl}$ gradient in the same buffer at a linear flow rate of $5 \mathrm{ml} \mathrm{min}^{-1}$. T4moD fractions were pooled based on activity and $\mathrm{T} 4 \mathrm{moC}$ was pooled based on the brown colour of the $[2 \mathrm{Fe}-$ 2S] cofactor. Purity of both proteins was monitored by SDS-PAGE. Pooled fractions were individually concentrated and applied to a Sephacryl S-100 $(45 \mathrm{~mm}$ diameter $\times 1,000 \mathrm{~mm}$, GE Health Care) column equilibrated in the above buffer at a linear flow rate of $3.33 \mathrm{ml} \mathrm{min}^{-1}$. Fractions were pooled based on activity and purity, concentrated to $\sim 1 \mathrm{mM}$ for both proteins, and exchanged into $10 \mathrm{mM}$ MOPS, pH 7.5, containing $50 \mathrm{mM} \mathrm{NaCl}$. The purified protein was drop frozen in liquid $\mathrm{N}_{2}$ and stored at $-80^{\circ} \mathrm{C}$.

Purification of T4moF. T4moF cell paste was re-suspended in $25 \mathrm{mM}$ MOPS, pH 7.5 , containing $80 \mathrm{mM} \mathrm{NaCl}$ and $2 \%$ glycerol at a ratio of $1.5 \mathrm{ml}$ per g of wet cell paste $^{55}$. The cell-free extract was prepared as described above for T4moH. The supernatant was carefully decanted and diluted with two volumes of the above buffer and loaded onto a DEAE Sepharose column $(45 \mathrm{~mm}$ diameter $\times 250 \mathrm{~mm}$, GE Health Care) equilibrated in the above buffer and eluted in an $80-400 \mathrm{mM}$ $\mathrm{NaCl}$ gradient in the same buffer at a flow rate of $5 \mathrm{ml} \mathrm{min}^{-1}$. T4moF was pooled based on the colour of the flavin adenine dinucleotide and [2Fe-2S] cofactors and catalytic activity. Purity was monitored by SDS-PAGE. Pooled fractions were concentrated and applied to a Sephacryl S-100 (45 mm diameter $\times 1,000 \mathrm{~mm}$, GE Health Care) column equilibrated with $25 \mathrm{mM}$ MOPS, pH 7.5, $200 \mathrm{mM} \mathrm{NaCl}$ and $5 \%$ glycerol at a flow rate of $3.33 \mathrm{ml} \mathrm{min}^{-1}$. Fractions were pooled based on activity and purity, concentrated to $\sim 1 \mathrm{mM}$ and exchanged into $10 \mathrm{mM}$ MOPS, $\mathrm{pH} 7.5$, containing $50 \mathrm{mM} \mathrm{NaCl}$. The purified protein was drop frozen in liquid $\mathrm{N}_{2}$ and stored at $-80^{\circ} \mathrm{C}$

Steady-state catalysis. Reactions with toluene were performed in $10 \mathrm{ml}$ crimp-sealed vials in $50 \mathrm{mM}$ sodium phosphate, $\mathrm{pH} 7.5$, containing $50 \mathrm{mM}$ $\mathrm{NaCl}, 3 \mathrm{mM} \mathrm{NADH}$, saturating substrate, $4 \mu \mathrm{M} \mathrm{T} 4 \mathrm{moH}, 0.4 \mu \mathrm{M}$ T4moF and varying amounts of $\mathrm{T} 4 \mathrm{moC}$ and $\mathrm{T} 4 \mathrm{moD}$. The reactions were quenched with $\mathrm{NaCl}$-saturated $2 \mathrm{~N} \mathrm{HCl}$, and extracted with chloroform containing 3-methyl benzylalcohol as an internal standard for toluene reactions. Products were quantified by gas chromatography using flame ionization detection (GD-FID) ${ }^{22}$.

Crystallization and structure determination. Crystals used to solve 4P1C were obtained by mixing $1.5 \mu \mathrm{l}$ of $\mathrm{T} 4 \mathrm{moH}(100 \mu \mathrm{M})$ and $1.5 \mu \mathrm{l}$ of $\mathrm{T} 4 \mathrm{moC}(200 \mu \mathrm{M})$ with an equal volume of $0.1 \mathrm{M}$ MOPS/HEPES, pH 7.5, containing 20\% PEG 3350, $200 \mathrm{mM}$ ammonium chloride, 5\% Jeffamine (Hampton Research) and $10 \mathrm{mM}$ $\mathrm{MgCl}_{2}$. After $\sim 1$ week at $19^{\circ} \mathrm{C}$, large thin petal-like crystals formed with dimensions $(\mu \mathrm{m})$ of $\sim 200 \times 500 \times 25$. Crystals used to solve $4 \mathrm{P} 1 \mathrm{~B}$ were obtained from hanging drop vapour diffusion by mixing $1.5 \mu \mathrm{l}$ of a mixture of $\mathrm{T} 4 \mathrm{moH}$ $(100 \mu \mathrm{M})$ and $\mathrm{T} 4 \mathrm{moC}(200 \mu \mathrm{M})$ with an equal volume of $0.1 \mathrm{M}$ Bis-Tris, $\mathrm{pH} 6.0$, containing 18\% PEG 3350, $200 \mathrm{mM}$ ammonium acetate, 5\% Jeffamine and $10 \mathrm{mM}$ $\mathrm{MgCl}_{2}$. After $\sim 1$ week at $19^{\circ} \mathrm{C}$, clusters of crystals were formed. Crystals of $4 \mathrm{P} 1 \mathrm{C}$ and $4 \mathrm{P} 1 \mathrm{~B}$ were cryo-protected in Fomblin 2500 and frozen in liquid $\mathrm{N}_{2}$. Diffraction data were collected at the Life Sciences Collaborative Access Team (LS-CAT) at the Advanced Photon Source (Argonne National Lab). Diffraction data for 4P1C were collected at station 21-ID-G $\lambda=0.97857 \AA$, whereas data for $4 \mathrm{P} 1 \mathrm{~B}$ were collected at station $21-\mathrm{ID}-\mathrm{D} \lambda=0.97857 \AA$, both at $100 \mathrm{~K}$. The data were indexed, integrated and scaled using HKL2000 (ref. 56). Phase solutions were obtained from CCP4 MolRep by using one protomer of T4moH PDB accession 3DHG as the starting model ${ }^{57}$. Electron density was fit and refined in multiple iterations using Phenix.refine ${ }^{57}$ and $\operatorname{Coot}^{58}$. Ramachandran and rotamer analysis were performed using MolProbity ${ }^{59}$. Ramachandran analysis (\%) for 4P1C resulted in 96.48 favourable, 3.42 allowed and 0.10 disallowed conformations, whereas analysis for 4P1B gave 97.0 favourable, 2.86 allowed and 0.16 disallowed conformations. Protein-protein interface surface areas were calculated using PISA ${ }^{60}$. Predictions of preferred electron transfer pathways were from HARLeM ${ }^{43}$. Figures were prepared using Pymol ${ }^{61}$

\section{References}

1. Jones, S. \& Thornton, J. M. Principles of protein-protein interactions. Proc. Natl Acad. Sci. USA 93, 13-20 (1996).

2. DeLano, W. L., Ultsch, M. H., de Vos, A. M. \& Wells, J. A. Convergent solutions to binding at a protein-protein interface. Science 287, 1279-1283 (2000).

3. Nordlund, P. \& Eklund, H. Di-iron-carboxylate proteins. Curr. Opin. Struct. Biol. 5, 758-766 (1995).

4. Lipscomb, J. D. Biochemistry of the soluble methane monooxygenase. Annu. Rev. Microbiol. 48, 371-399 (1994).

5. Tinberg, C. E. \& Lippard, S. J. Dioxygen activation in soluble methane monooxygenase. Acc. Chem. Res. 44, 280-288 (2011).

6. Hanson, R. S. \& Hanson, T. E. Methanotrophic bacteria. Microbiol. Rev. 60, 439-471 (1996).

7. Teufel, R., Friedrich, T. \& Fuchs, G. An oxygenase that forms and deoxygenates toxic epoxide. Nature 483, 359-362 (2012)

8. Wallar, B. J. \& Lipscomb, J. D. Dioxygen activation by enzymes containing binuclear non-heme iron clusters. Chem. Rev. 96, 2625-2658 (1996).

9. Makris, T. M., Chakrabarti, M., Munck, E. \& Lipscomb, J. D. A family of diiron monooxygenases catalyzing amino acid beta-hydroxylation in antibiotic biosynthesis. Proc. Natl Acad. Sci. USA 107, 15391-15396 (2010).

10. Fox, B. G., Lyle, K. S. \& Rogge, C. E. Reactions of the diiron enzyme stearoylacyl carrier protein desaturase. Acc. Chem. Res. 37, 421-429 (2004).

11. Shanklin, J., Guy, J. E., Mishra, G. \& Lindqvist, Y. Desaturases: emerging models for understanding functional diversification of diiron-containing enzymes. J. Biol. Chem. 284, 18559-18563 (2009).

12. Stubbe, J. Di-iron-tyrosyl radical ribonucleotide reductases. Curr. Opin. Chem Biol. 7, 183-188 (2003).

13. Sahlin, M. \& Sjoberg, B. M. Ribonucleotide reductase. A virtual playground for electron transfer reactions. Subcell. Biochem. 35, 405-443 (2000).

14. Tinberg, C. E., Song, W. J., Izzo, V. \& Lippard, S. J. Multiple roles of component proteins in bacterial multicomponent monooxygenases: phenol hydroxylase and toluene/o-xylene monooxygenase from Pseudomonas sp. OX1. Biochemistry 50, 1788-1798 (2011).

15. Paulsen, K. E. et al. Oxidation-reduction potentials of the methane monooxygenase hydroxylase component from Methylosinus trichosporium OB3b. Biochemistry 33, 713-722 (1994). 
16. Hendrich, M. P., Münck, E., Fox, B. G. \& Lipscomb, J. D. Integer-spin EPR studies of the fully reduced methane monooxygenase hydroxylase component. J. Am. Chem. Soc. 112, 5861-5865 (1990).

17. Fox, B. G., Liu, Y., Dege, J. E. \& Lipscomb, J. D. Complex formation between the protein components of methane monooxygenase from Methylosinus trichosporium OB3b. Identification of sites of component interaction. J. Biol. Chem. 266, 540-550 (1991).

18. Hendrich, M. P., Fox, B. G., Andersson, K. K., Debrunner, P. G. \& Lipscomb, J. D. Ligation of the diiron site of the hydroxylase component of methane monooxygenase. An electron nuclear double resonance study. J. Biol. Chem. 267, 261-269 (1992).

19. Liu, K. E. et al. Kinetic and spectroscopic characterization of intermediates and component interactions in reactions of methane monooxygenase from Methylococcus capsulatus (Bath). J. Am. Chem. Soc. 117, 10174-10185 (1995).

20. Mitic, N., Schwartz, J. K., Brazeau, B. J., Lipscomb, J. D. \& Solomon, E. I. CD and MCD studies of the effects of component B variant binding on the biferrous active site of methane monooxygenase. Biochemistry 47, 8386-8397 (2008).

21. Green, J. \& Dalton, H. Protein B of soluble methane monooxygenase from Methylococcus capsulatus (Bath). A novel regulatory protein of enzyme activity. J. Biol. Chem. 260, 15795-15801 (1985).

22. Mitchell, K. H., Studts, J. M. \& Fox, B. G. Combined participation of hydroxylase active site residues and effector protein binding in a para to ortho modulation of toluene 4-monooxygenase regiospecificity. Biochemistry 41, 3176-3188 (2002).

23. Wallar, B. J. \& Lipscomb, J. D. Methane monooxygenase component B mutants alter the kinetics of steps throughout the catalytic cycle. Biochemistry 40, 2220-2233 (2001).

24. Liu, Y., Nesheim, J. C., Lee, S. K. \& Lipscomb, J. D. Gating effects of component $\mathrm{B}$ on oxygen activation by the methane monooxygenase hydroxylase component. J. Biol. Chem. 270, 24662-24665 (1995).

25. Liu, Y., Nesheim, J. C., Paulsen, K. E., Stankovich, M. T. \& Lipscomb, J. D. Roles of the methane monooxygenase reductase component in the regulation of catalysis. Biochemistry 36, 5223-5233 (1997).

26. Banerjee, R., Meier, K. K., Munck, E. \& Lipscomb, J. D. Intermediate $\mathrm{P}^{*}$ from soluble methane monooxygenase contains a diferrous cluster. Biochemistry 52, 4331-4342 (2013)

27. Froland, W. A., Andersson, K. K., Lee, S. K., Liu, Y. \& Lipscomb, J. D. Methane monooxygenase component $\mathrm{B}$ and reductase alter the regioselectivity of the hydroxylase component-catalyzed reactions. A novel role for protein-protein interactions in an oxygenase mechanism. J. Biol. Chem. 267, 17588-17597 (1992).

28. Bailey, L. J., McCoy, J. G., Phillips, Jr G. N. \& Fox, B. G. Structural consequences of effector protein complex formation in a diiron hydroxylase. Proc. Natl Acad. Sci. USA 105, 19194-19198 (2008).

29. Elsen, N. L., Bailey, L. J., Hauser, A. D. \& Fox, B. G. Role for threonine 201 in the catalytic cycle of the soluble diiron hydroxylase toluene 4-monooxygenase. Biochemistry 48, 3838-3846 (2009).

30. Bailey, L. J. \& Fox, B. G. Crystallographic and catalytic studies of the peroxide-shunt reaction in a diiron hydroxylase. Biochemistry 48, 8932-8939 (2009).

31. Bailey, L. J. et al. Crystallographic analysis of active site contributions to regiospecificity in the diiron enzyme toluene 4-monooxygenase. Biochemistry 51, 1101-1113 (2012)

32. Leahy, J. G., Batchelor, P. J. \& Morcomb, S. M. Evolution of the soluble diiron monooxygenases. FEMS Microbiol. Rev. 27, 449-479 (2003).

33. Behan, R. K. \& Lippard, S. J. The aging-associated enzyme CLK-1 is a member of the carboxylate-bridged diiron family of proteins. Biochemistry 49, 9679-9681 (2010).

34. Pikus, J. D. et al. Recombinant toluene-4-monooxygenase: catalytic and Mossbauer studies of the purified diiron and rieske components of a fourprotein complex. Biochemistry 35, $9106-9119$ (1996).

35. Elsen, N. L., Moe, L. A., McMartin, L. A. \& Fox, B. G. Redox and functional analysis of the Rieske ferredoxin component of the toluene 4-monooxygenase. Biochemistry 46, 976-986 (2007).

36. Moe, L. A., Bingman, C. A., Wesenberg, G. E., Phillips, Jr G. N. \& Fox, B. G. Structure of T4moC, the Rieske-type ferredoxin component of toluene 4-monooxygenase. Acta. Crystallogr. D. Biol. Crystallogr. 62, 476-482 (2006).

37. McCormick, M. S., Sazinsky, M. H., Condon, K. L. \& Lippard, S. J. X-ray crystal structures of manganese(II)-reconstituted and native toluene/o-xylene monooxygenase hydroxylase reveal rotamer shifts in conserved residues and an enhanced view of the protein interior. J. Am. Chem. Soc. 128, 15108-15110 (2006).

38. Rosenzweig, A. C., Frederick, C. A., Lippard, S. J. \& Nordlund, P. Crystal structure of a bacterial non-haem iron hydroxylase that catalyses the biological oxidation of methane. Nature 366, 537-543 (1993).

39. Sazinsky, M. H., Bard, J., Di Donato, A. \& Lippard, S. J. Crystal structure of the toluene/o-xylene monooxygenase hydroxylase from Pseudomonas stutzeri OX1.
Insight into the substrate specificity, substrate channeling, and active site tuning of multicomponent monooxygenases. J. Biol. Chem. 279, 30600-30610 (2004).

40. Schulenburg, C. \& Hilvert, D. Protein conformational disorder and enzyme catalysis. Top Curr. Chem. 337, 41-67 (2013).

41. Moser, C. C., Keske, J. M., Warncke, K., Farid, R. S. \& Dutton, P. L. Nature of biological electron transfer. Nature 355, 796-802 (1992).

42. Gray, H. B. \& Winkler, J. R. Electron transfer in proteins. Annu. Rev. Biochem. 65, 537-561 (1996)

43. Kurnikov, I. V. HARLEM molecular modelling package (Department of Chemistry, University of Pittsburgh, 2000).

44. Song, W. J. et al. Active site threonine facilitates proton transfer during dioxygen activation at the diiron center of toluene/o-xylene monooxygenase hydroxylase. J. Am. Chem. Soc. 132, 13582-13585 (2010).

45. Whittington, D. A. \& Lippard, S. J. Crystal structures of the soluble methane monooxygenase hydroxylase from Methylococcus capsulatus (Bath) demonstrating geometrical variability at the dinuclear iron active site. J. Am. Chem. Soc. 123, 827-838 (2001).

46. McCormick, M. S. \& Lippard, S. J. Analysis of substrate access to active sites in bacterial multicomponent monooxygenase hydroxylases: X-ray crystal structure of xenon-pressurized phenol hydroxylase from Pseudomonas sp. OX1. Biochemistry 50, 11058-11069 (2011).

47. Sevrioukova, I. F. Redox-dependent structural reorganization in putidaredoxin, a vertebrate-type [2Fe-2S] ferredoxin from Pseudomonas putida. J. Mol. Biol. 347, 607-621 (2005).

48. Tripathi, S., Li, H. \& Poulos, T. L. Structural basis for effector control and redox partner recognition in cytochrome P450. Science 340, 1227-1230 (2013).

49. Arnold, K., Bordoli, L., Kopp, J. \& Schwede, T. The SWISS-MODEL workspace: a web-based environment for protein structure homology modelling. Bioinformatics 22, 195-201 (2006).

50. Colbert, C. L., Couture, M. M., Eltis, L. D. \& Bolin, J. T. A cluster exposed: structure of the Rieske ferredoxin from biphenyl dioxygenase and the redox properties of Rieske Fe-S proteins. Structure 8, 1267-1278 (2000).

51. Lee, S. K., Nesheim, J. C. \& Lipscomb, J. D. Transient intermediates of the methane monooxygenase catalytic cycle. J. Biol. Chem. 268, 21569-21577 (1993).

52. Zhang, J. et al. Methane monooxygenase hydroxylase and B component interactions. Biochemistry 45, 2913-2926 (2006).

53. Wang, W., Iacob, R. E., Luoh, R. P., Engen, J. R. \& Lippard, S. J. Electron transfer control in soluble methane monooxygenase. J. Am. Chem. Soc. 136, 9754-9762 (2014).

54. Fox, B. G. \& Blommel, P. G. Autoinduction of protein expression. Curr. Protoc. Protein. Sci. Chapter 5, Unit 523 (2009).

55. Bailey, L. J., Elsen, N. L., Pierce, B. S. \& Fox, B. G. Soluble expression and purification of the oxidoreductase component of toluene 4-monooxygenase. Protein Expr. Purif. 57, 9-16 (2008).

56. Otwinowski, Z. \& Minor, W. Processing of X-ray diffraction data collected in oscillation mode. Macromol. Crystallogr. A 276, 307-326 (1997).

57. Vagin, A. \& Teplyakov, A. Molecular replacement with MOLREP. Acta. Crystallogr. D. Biol. Crystallogr. 66, 22-25 (2010).

58. Emsley, P. \& Cowtan, K. Coot: model-building tools for molecular graphics. Acta. Crystallogr. D. Biol. Crystallogr. 60, 2126-2132 (2004).

59. Chen, V. B. et al. MolProbity: all-atom structure validation for macromolecular crystallography. Acta Crystallogr. D Biol. Crystallogr. 66, 12-21 (2010).

60. Krissinel, E. \& Henrick, K. Inference of macromolecular assemblies from crystalline state. J. Mol. Biol. 372, 774-797 (2007).

61. DeLano, W. L. The PyMOL Molecular Graphics System (DeLano Scientific, 2002).

\section{Acknowledgements}

This work was funded by the National Science Foundation MCB-0843239 to B.G.F. Use of the Advanced Photon Source was supported by the US Department of Energy, Office of Science contract No. W-31-109-ENG-38. Use of the Life Science Collaborative Access Team (LS-CAT) was supported by the College of Agricultural and Life Sciences, Department of Biochemistry, and Graduate School of the University of Wisconsin.

\section{Author contributions}

J.F.A designed experiments, obtained crystals, solved and refined structures, analysed data and wrote the manuscript. L.J.B. designed experiments, obtained initial crystallization results and analysed data. N.L.E. designed exeriments, performed enzymatic assays and analysed data. B.G.F. designed experiments, analysed data and wrote the manuscript. All authors discussed results and commented on the manuscript. 


\section{Additional information}

Accession codes: Atomic coordinates and structure factors have been deposited in the Protein Databank under the accession codes $4 \mathrm{P} 1 \mathrm{~B}$ and $4 \mathrm{P} 1 \mathrm{C}$, respectively.

Supplementary Information accompanies this paper at http://www.nature.com/ naturecommunications

Competing financial interests: The authors declare no competing financial interests.

Reprints and permission information is available online at http://npg.nature.com/ reprintsandpermissions/
How to cite this article: Acheson, J. F. et al. Structural basis for biomolecular recognition in overlapping binding sites in a diiron enzyme system. Nat. Commun. 5:5009 doi: $10.1038 /$ ncomms6009 (2014)

(c) (i) This work is licensed under a Creative Commons Attribution 4.0 International License. The images or other third party material in this article are included in the article's Creative Commons license, unless indicated otherwise in the credit line; if the material is not included under the Creative Commons license, users will need to obtain permission from the license holder to reproduce the material. To view a copy of this license, visit http://creativecommons.org/licenses/by/4.0/ 